\title{
TUMORES FUNCIONALES DEL OVARIO
}

\author{
Prof. Rubén Darío Guzmán A. \\ Prof. Alvaro Fonnegra $M$. \\ Prof. Ricardo Alvarado P. \\ Dr. Armando Moreno
}

\section{Conceptos generales:}

Siguiendo los conceptos modernos de Norris y Chorlton (21) los Tumores Funcionales del Ovario pueden dividirse en varios grupos, el más amplio de los cuales deriva del estroma gonadal especializado: pertenecen a este grupo los Tecomas, los Tumores de Células de la Granulosa, de Sertoli-Leydig o arrenoblastoma y los Tumores no específicos del estroma gonadal. En este grupo hay que incluir los Tumores de Células Lípidas, sea cuales fueren sus características histológicas y aquellos de localización en el hilio del ovario, ya que se desconoce de una manera cierta el origen de las células hiliares.

1-TUMORES DEL ESTROMA GONA-

DAL:

Tumor de células granulosas y

Tecoma

Tumor de Sertoli-Leydig

(arrenoblastoma)

Tumor de Sertoli

(foliculoma lípido)

Tumores de células lípidas

2-TUMORES CON ESTROMA

FUNCIONAL:

Tumor de Brenner

Adenofibroma

Cistadenoma
Cistadenocarcinoma

Carcinoma metastásico

3-TUMORES FUNCIONALES DE CELULAS GERMINATIVAS:

Coriocarcinoma

Teratoma con componentes endocrinos

4-TUMORES MIXTOS DEL ESTROMA Y DE CELULAS GERMINATIVAS Gonadoblastoma

\section{5-TUMORES NO ENDOCRINOS} CON EFECTO ENDOCRINO

6-HIPERPLASIAS TUMORALES QUE SIMULAN NEOPLASIAS: Hiperplasia nodular luteínica de la teca en el embarazo Hiperplasia de células del hilio.

Consideran estos mismos autores que toda terminología o clasificación basada en una teoría histológica del origen celular es inestable y puede quedar rápidamente anticuada al surgir nuevos conocimientos, lo que resulta cierto referente a los tumores del ovario donde el origen embriológico de las células y líneas celulares es confuso e incierto; hasta el momento no existe certeza alguna respecto al origen de la mayor parte de las estructuras ováricas del adulto, ya que no ha sido posible la observación 
prolongada de las células en el curso de sus cambios de tamaño, forma y posición durante la evolución de la gónada, por lo que se ha perdido su identidad y hasta quizá hayan cambiado su función. A pesar de estas dificultades los tumores mesenquimatosos pueden dividirse conceptualmente en dos tipos: Aquellos que poseen función endocrina potencial y los que carecen de la misma. Los que poseen células con capacidad para producir esteroides son cuando menos potencialmente funcionales, aquí están incluídos los tecomas, tumores de células de la granulosa y los arrenoblastomas y los que no poseen función endocrina potencial y en consecuencia estas neoplasias derivan de las estructuras y células puramente de sostén como fibroblastos y vasos sanguíneos que no tienen capacidad esteroidógena dando origen a tumores como fibromas, hemangiomas $y$ leiomiomas. Los tumores del estroma gonadal difieren básicamente de los otros tumores mesenquimatosos del ovario por poseer capacidad de producir esteroides hormonales activos ya sean androgénicos o estrogénicos según sus manifestaciones funcionales. El 10\% aproximadamente de los tumores del estroma gonadal no pueden clasificarse con certeza en ningún grupo específico por lo que han recibido el nombre de tumores de tipo inespecífico. Es bueno aclarar que el tumor de Sertoli-Leydig también se conoce con los nombres de arrenoblastoma y es el tumor virilizante más frecuente del ovario; el tumor de Sertoli es una variedad rara del tumor del estroma gonadal compuesto casi enteramente de columnas y cordones de células parecidas a las de Sertoli y dispuestas en tal forma que recuerdan túbulos seminíferos. Raro en la mujer, es muy frecuente en los caninos y bovinos; en todas es- tas últimas especies tiende a producir estrógenos.

Los tumores de Células Lípidas son tumores clínica e histológicamente similares a los incluídos bajo las designaciones de Tumor de Células del hilio, tumor de residuos de la córticosuprarrenal, masculinoblastoma etc.

Los tumores no específicos del estroma gonadal la mayor parte son tumores primitivos incompletamente diferenciados o con diferenciación bifásica hacia estructuras testiculares $u$ ováricas, hasta el punto de que la neoplasia no se adapta a ninguna de las categorías del estroma definitivo. Se ha empleado el término de ginandroblastoma para referirse a estos tumores inespecíficos del estroma $y$ pueden tener manifestaciones funcionales asociadas feminizantes, virilizantes y ocasionalmente de ambos tipos.

Se ha dicho que los tumores primarios y metastásicos del ovario poseen función hormonal aunque rara $v \in z$ se observan tales efectos; el tumor propiamente dicho no elabora hormonas las cuales proceden más bien de las células del estroma ovárico cercana al tumor y mezcladas con el mismo.

Referente a los tumores funcionales de células germinativas podemos decir que existe el Coriocarcinoma primario del ovario que es muy raro y los teratomas con actividad endocrina, como es el estroma ovárico.

En cuanto a los tumores no endocrinos con efectos endocrinos se refiere a una situación clínica en la cual la neoplasia benigna o maligna, nacida de un tejido no endocrino se acompaña de signos evidentes clínicos y de laboratorio de producción de algún tipo hormonal, según Goodall (10). Se ha informado de neoplasias 
ováricas asociadas con síndromes paraendocrinos como hipercalcemia, hipertiroidismo, hipoglicemia, eritemia y síndrome de Cushing. Algunos estudios se han referido también a la producción de gonadotropina y prolactina.

Entre las hiperplasias tumorales que simulan neoplasias están la hiperplasia nodular luteinizante de la teca de la gestación y la hiperplasia de las células del hilio, la cual puede presentarse en el nacimiento, pubertad, después de la menopausia y de irradiación o administración de gonadotropinas y en paciente con coriocarcinoma y embarazo ectópico.

La hipertecosis y ciertos síndromes relacionados con el de Stein-Leventhal pueden acompañarse de tal virulización que plantean la posibilidad de un tumor ovárico funcional.

\section{Tumores de las células granulosas Teca Granulosas - Tecomas:}

Representan el $3 \%$ al $6 \%$ de los tumores ováricos y casi el $80 \%$ de los tumores del estroma gonadal (21). Su frecuencia varía de un informe a otro pero puede llegar a un $9 \%$ de todos los tumores ováricos en algunas instituciones. Según Kottmeier (14) los tumores granulo-tecales combinados constituyen el $4 \%$ al $9 \%$ de todas las neoplasias ováricas. Hertig y Gore (13) observaron que la incidencia en el Boston Hospital for women era aproximadamente del $6 \%$; también observaron una distribución de los diversos tipos; células de la granulosa; $17,5 \%$; células granulotecales: $15 \%$ y células tecales: $67,5 \%$. Según Benson los tumores de las células de la granulosa represen$\tan$ el $3 \%$ al $4 \%$. Según Griffiths (12) los tecomas no son tan comunes como los de la granulosa y constituyen $\tan$ solo el $1 \%$ al $2 \%$ de todas las neoplasias ováricas.
En nuestro medio, según Goubert (11), la frecuencia es de 0,71\% para los tumores de células de la granulosa, igual frecuencia para los tecomas, entre todos los tumores ováricos, en una revisión de todas las neoplasias comprendidas entre el año 1953 a 1960 en el Hospital San Juan de Dios de Bogotá.

Es frecuente utilizar el término de Foliculoma lipídico para aquellos tumores de células granulosas muy luteinizados. López Escobar (15) los llama Tumores displásicos.

\section{Origen :}

El origen de estos tumores no es muy claro. Según Griffiths (12) las células de la granulosa normal y las células de la teca interna y de la teca externa se originan en el estroma cortical ovárico si se presenta un óvulo como "organizador celular". Hertig y Gore (13) y Novak (22) creen que estos tumores se producen a partir del estroma ovárico o de sus derivados de la pared folicular. Norris y Chorlton (21) también consideran que se derivan del estroma gonadal. Otras teorías de histogénesis incluyen la de Gilman, citado por Griffiths (12), el cual sugiere que las células granulosas se derivan del epitelio germinal y las células tecales del estroma cortical. Robert Meyer (19) opina que estos tumores se engendran a partir de "granulosa ballen" y los considera restos de células granulosas en la corteza ovárica. Sternberg y Caskill, citados por Griffiths (12) han trazado la génesis de los tecomas a partir de la hiperplasia estromocortical mientras que Traut, Butterworth citado por Griffiths y McKay (18) colabs. citados por el mismo autor anterior, conceptúan que los tumores de células de la granulosa se derivarían de restos del epitelio folicular en folículos 
atrésicos y los tecomas de una hiperplasia cortical del estroma. Según Woll y cols., citado por este último autor (12), la histogénesis de los tecomas difiere de la de los tumores de células de granulosa, pues los primeros se originan de una hiperplasia estromocortical. Este concepto se basa: 1․ El patrón histológico de ambos tejidos es idéntico, incluyendo la presencia de placas hialinas, 2 . Se observan etapas intermedias entre las hiperplasias estromocorticales y los tecomas, $3^{\circ}$. Las pacientes que sufren tumores de células de la teca en un ovario, tienen, de manera invariable, hiperplasia estromocortical y $4{ }^{\circ}$ Ambos procesos se producen principalmente en la post-menopausia.

El posible origen de estas neoplasias en restos embrionarios ha recibido muy poca aceptación por la mayoría de los autores.

\section{Anatomía Patológica:}

Estos tumores varían de tamaño descle unos pocos milímetros de diámetro hasta formaciones de un tamaño bastante considerable. Los tumores de células de granulosa cuando son pequeños son sólidos pero los más voluminosos presentan a menudo una o varias cavidades quísticas. El tejido sólido que entra en su formación tiene consistencia friable 0 granulosa y es de color grisáceo y a veces amarillento. Al hacer el corte los tumores de granulosa se identifican zonas de hemorragia, necrosis o licuefacción.

El aspecto microscópico es interesante, pues se basa en el carácter granuloso de las células constitutivas $y$ en los caracteres de desarrollo de las mismas. Presentan cierta tendencia a la formación de diminutas áreas quísticas de licuación que corresponde a los cuerpos de Call-Exner tan típicos de la granulosa, sobre todo en roedores como el conejo. Las células de la granulosa son de tamaño uniforme y de forma poligonal con límites citoplasmáticos poco delimitados. El protoplasma es ligeramente granular y eosinófilo; los núcleos son grandes, ovoides o redondos, de homogénea tinción y con escasísimas mitosis. Cuando existe buena cantidad de componente tecal puede observarse como estas células tecales son fusiformes, tienen núcleos ovoides y parecen fibroblastos. Los depósitos grasos de las células tecales pueden destacarse mediante tinciones específicas para diferenciar a éstos de los fibroblastos. Las células tecales pueden ser diferenciadas de las de los tumores de la granulosa en algunos tumores más anaplásicos mediante la utilización de tinciones del retículo, ya que alrededor de las primeras se observa siempre un retículo de sostenimiento, cosa que no sucede en las células individuales de la granulosa.

Histológicamente se han identificado numerosos patrones de los tumores de células de la granulosa, conocidos como variables: foliculoide, adenomatoide, cilindroide, sarcomatoide y trabeculoide. El patrón más corriente es el foliculoide, en el que las células se disponen en rosetas con núcleos situados en ángulos rectos a un espacio central. Esta disposición recuerda la del folículo en desarroIlo. Si el patrón es muy acentuado hasta el punto de formarse nódulos glandulares, la variedad recibe el nombre de adenomatoide, mientras que si las células se organizan de manera difusa estamos ante una variante sarcomatoide. Si las células de la granulosa se disponen en columnas bastante típicas con las células tecales organizadas en láminas o acúmulos en torno a esas columnas celulares estamos ante la variedad cilin- 
droide. Cuando se produce la luteinización, las células aumentan de tamaño, se destacan más sus perfiles y se vacuolizan.

En cuanto al cuadro histológico del tecoma, vemos como el elemento conjuntivo puede conferir a la imagen histológica un aspecto semejante al fibroma. Los tumores de la teca son en general firmes y fibrosos aunque al igual que los tumores de la granulosa pueden presentar cierta tendencia a la degeneración quística.

Microscópicamente se encuentran paquetes de células fusiformes anchas, de aspecto epiteloide distribuídos en una forma entrelazada e irregular por todo el tumor, separado por bandas de tejido conjuntivo y placas hialinas de tamaño variable. También se insiste en la presencia de gran cantidad de grasa birrefrigente dentro de las células y en menor cantidad en el tejido conjuntivo circundante. Las tinciones de lipoides muestran la presencia de grandes cantidades de grasa birrefrigente, casi siempre intracelular, esto es muy sugestivo pero no patognomónico de actividad esteroide. Frecuentemente se observan áreas que parecen células netamente granulosas de manera que cabe dudar de la conveniencia de una división demasiado neta entre tumores de la granulosa y tecales, especialmente si se tienen en cuenta sus efectos endocrinos análogos, por lo tanto este tumor no debe considerarse como un tumor específico constituído solamente por células de la teca, ya que si se realizan cortes suficientes, se descubren cierto número de células de la granulosa. Suele aceptarse que la producción hormonal procede de las células tecales activas, las cuales contienen una sustancia llamada quitona la cual se hace presente mediante el empleo de métodos histoquímicos, suponiéndose según al- gunos autores que constituye la localización de la síntesis esteroidea.

\section{Malignidad:}

Ackerman L. y Del Regato Juan (1) consideran los tumores de la granulosa potencialmente malignos. Parsons y Sommers (23) afirman que más del $30 \%$ de estos tumores tienen una tendencia a la malignidad. Robbins (24) y Botella (4) consideran que el $80 \%$ de los tumores de la granulosa son benignos y el $20 \%$ malignos. Según Griffiths (12) el tumor de células de la granulosa es poseedor de un bajo potencial de malignidad ( $3 \%-4 \%)$, pero la tasa de recidiva puede elevarse hasta un $25 \%$ o un $30 \%$. Algunos de estos tumores son histológicamente benignos, pero malignos desde el punto de vista clínico, ya que pueden observarse recidivas locales en número considerable en un lapso de 10 a 15 años. Sin embargo, puede producirse un tipo de tumor de células de la granulosa con carácter decididamente maligno y que posee todos los caracteres histológicos del carcinoma; en estos tumores las células de la teca suelen permanecer benignas en apariencia y las metástasis de tal lesión pueden realizarse a través de los vasos linfáticos y por vía hemática. En una revisión de 96 pacientes de los archivos del registro de Tumores Ováricos, Busbey y Anderson (5) encuentran una incidencia del $25 \%$ de malignidad. Jones y Telinde citados por Novak (22), Sommers, Gates y Goodof (25) han observado recidivas tardías después de 20 años. Flick y Banfield (9) indican que el tecoma solo tiene el tercio de malignidad con relación a la malignidad de los tumores de las células de la granulosa, igual opinión comparte Novak (22). Diddle A. W. (6) dan un índice de recurrencia hasta del $28 \%$ pero en los trabajos de Norris y Chorlton (21) observaron 
$\tan$ solo un $7 \%$ de recurrencias cuando se trataba de tumores de la granulosa. El concenso general de todos los autores es que los tumores de la granulosa tienen más potencial de malignidad que los tumores de la teca. Según Norris y Taylor (20) son muy raros los tecomas malignos publicados y en muchos de ellos cabe dudar de su diagnóstico. Algunos tecomas malignos compuestos de células hipercromáticas pleomórficas con gran actividad mitótica merecen más bien el nombre de sarcoma. Según estos últimos autores para calificar una neoplasia como tecoma maligno es preciso comprobar actividad funcional, comportamiento propio de un tumor maligno y además ser descubierto cuando se halla limitado al ovario para eliminar la confusión de otros sarcomas localizados en la región de este órgano como el fibrosarcoma ovárico primario, o de otros órganos como el fibrosarcoma retroperitoneal, mesotelioma y leiomiosarcoma del útero o de los tejidos de la pelvis. En la literatura mundial existen menos de 20 tecomas malignos que bien podrían tratarse de ovarios con tumores de células de la granulosa de variedad sarcomatoide. Ahora bien, como generalmente existe una combinación de componentes epiteloides y de tejido que parece conectivo, resulta difícil la afirmación precisa sobre la malignidad de estos últimos tumores. Según Benson (3) los tecomas tienen una incidencia muy baja de malignidad: $1 \%$ y por lo tanto el pronóstico es excelente después de la cirugía, en cambio la incidencia de malignidad de los tumores de la granulosa alcanza entre el $15 \%$ al $20 \%$.

También es importante comentar sobre al asociación de los tumores feminizantes con $\mathrm{Ca}$. de endometrio, así varios investigadores (22) han encon- trado que las mujeres postmenopáusicas con tumores feminizantes desarrollan $\mathrm{Ca}$. endometrial en el $15 \%$ al $20 \%$ de los casos. Dockertey y Mussey, citados por Griffiths (12) en un estudio realizado de tumores de la granulosa encontraron una incidencia del $15 \%$ de $\mathrm{Ca}$. endometrial; Emge (8), sólo encontró un 3,12\% y Grady, un porcentaje parecido. Hertig y Gore (13) en un estudio de 75 tumores mesenquimatosos feminizantes encontró que un $60 \%$ mostraba una hiperplasia endometrial y una cuarta parte de $\mathrm{Ca}$. de endometrio. Según Norris y Chorlton (21) existe hiperplasia endometrial en el $2 \%$ y según Mansell y Hertig (16) Matz (19) existe Ca. endometrial en un 15\%. Duarte Contreras (7) presenta un caso de tumor de granulosa asociado a Ca. de endometrio ocurrido en el Hospital San Juan de Dios de Cúcuta durante los años de 1963 a 1967. Parece que el tecoma ejerce efecto carcinógeno más frecuente sobre el endometrio que los demás tumores funcionantes.

\section{Material y Método:}

Se presentan 7 casos de tumores de células de granulosas, 2 casos de tumores Teca-granulosa y 3 casos de Tecomas de ovarios hospitalizados y tratados en el servicio de Ginecología del Instituto Materno Infantil de Bogotá "Concepción Villaveces de Acosta" en un lapso comprendido entre el 1\% de Enero de 1960 y el 30 de Junio de 1975, es decir, 15 años $y$ medio.

Todos estos tumores pueden presentarse en cualquier edad, pudiéndose encontrar en mortinatos y en mujeres de edad avanzada (20). Según Griffiths (12) la mayor parte de los tumores de la granulosa se presentan durante los años postmenopáusicos y sólo unos pocos en la época 


\begin{tabular}{cccccc}
\hline Edad & & Granulosa & Teca-Granulosa & Tecoma \\
\hline $12-30$ años & 45 años & 2 & & \\
$31-60$ años & 2 & 1 & 1 \\
$46-75$ años & 2 & 1 & - \\
$61-6$ & -7 & 2 & 3 \\
\hline
\end{tabular}

prepuberal. En un estudio de Hodgson, citado por Griffiths (12), la edad promedio es de 52 años, un $61 \%$ suele ocurrir en la edad postmenopáusica; un $37 \%$ en la vida sexual activa y algo menos del $20 \%$ antes de la pubertad. Según Norris y Taylor (20) la edad promedio de las pacientes es de 42 años, ya sea que se trate de tumores de la granulosa o de la teca. El tecoma según Benson (3), puede encontrarse en cual- quier edad pero se sabe que el $70 \%$ se produce en la postmenopausia.

En nuestro estudio no puede concluirse en una forma precisa en cuanto a edad se refiere debido al número $\tan$ escaso de casos estudiados. La paciente más joven fue de 24 años con un tumor de la granulosa y la de mayor edad de 70 años con un tumor Tecagranulosa.

\begin{tabular}{lccc}
\hline Sintomatología & Granulosa & Teca-Granulosa & Tecoma \\
Masa abdominal & & 4 & 2 \\
Masa abdominal, dolor hipogástrico & 1 & 1 & 1 \\
Masa abdominal, hemorragia genital & 1 & -1 & 3 \\
Hemorragia genital & 7 & 3
\end{tabular}

La sintomatología de estos tumores depende de la actividad estrogénica y la época en que se presenten estos tumores: prepuberal, actividad sexual y postmenopausia. Se acepta, que el grado de actividad hormonal en cada tumor es variable y como dijimos anteriormente las características clínicas que se producen como resultado de la secreción de estrógenos variarán en relación con la edad de la paciente, así en la muchacha impúber se producirá el síndrome de seudopubertad precoz, caracterizado por el desarrollo de un contorno femenino adulto, crecimiento mamario, vello pubiano y axilar, agrandamiento de los genitales externos e internos, hemorragia anovulatoria, endo- metrio hiperplásico y frotis vaginal estrogénico. Durante el período postpuberal, el cuadro clínico no es tan llamativo pero si el tumor produce marcada secreción estrogénica son frecuentes las hemorragias irregulares; puede suprimirse la ovulación y la enferma entrar en amenorrea. Cuando el tumor se presenta en el período postmenopáusico sus síntomas serán de hemorragias uterinas irregulares, en ocasiones hipertrofia mamaria. Existen casos muy raros de tumores de estos tipos que en lugar de producir feminización produce virilización. También estos tumores pueden dar una sintomatología igual a la que puede producir cualquier tumor ovárico: presencia de masa 
pélvica, dolor pélvico por compresión sobre los órganos vecinos y aún pueden complicarse con fenómenos de torsión. Es bueno anotar que en la mayoría de los tecomas los efectos clínicos son variables y algunos pueden carecer de síntomas y en otros los síntomas son muy acentuados. Se ha comprobado en los tecomas la presencia de miomas uterinos así como de la de Carcinoma de endometrio simultáneo. Es factible que se presente al igual que en los tumores de células de granulosa, el síndrome de Meigs (ascitis e hidrotórax). Un caso por nosotros estudiado se encontró un derrame pleural pero el líquido fue positivo para células tumorales.

\section{Diagnóstico preoperatorio:}

En ningún caso se hizo un diag- nóstico definitivo preoperatorio de estos tumores. Los diagnósticos preoperatorios fueron de miomatosis uterina, quiste de ovario y de Ca. de ovario etc. Se practicaron exámenes preoperatorios de Urografía, Radiografía de tórax, colon por enema que nos mostraban la existencia de una masa pélvica que en algunas ocasiones rechazaban el colon. En todos los casos se practicó citología vaginal funcional y tumoral pero que no llegaron a concluir nada definitivo sobre el funcionalismo de estos tumores. En algunos casos se efectuó raspado uterino cuyo resultado varió desde un endometrio atrófico hasta una hiperplasia glandulo-quística $\mathrm{y} / \mathrm{o}$ una adenomatosa; no se encontró ningún caso de Ca. endometrial.

Todos estos tumores generalmente son unilaterales. Según Novak (22)

\begin{tabular}{lccc}
\hline Laparotomía & Granulosa & Teca-Granulosa & Tecoma \\
\hline Ovario derecho & 3 & & 2 \\
Ovario izquierdo & 1 & 1 & 1 \\
Bilateral & 3 & 1 & - \\
& 7 & 2 & 3 \\
\hline
\end{tabular}

la bilateralidad es rara encontrándose aproximadamente en el $5 \%$ de los casos. Griffiths (12) da un porcentaje de bilateralidad del $12 \%$ al $18 \%$. Anderson (2) da un porcentaje de bilateralidad del $12 \%$ al $17,5 \%$. En nuestros casos no se puede concluir nada diciente por el escaso número de ellos. En muy pocos casos existía ascitis, invasión a órganos vecinos como peritoneo, colon sigmoides, intestino delgado y epiplón.

\begin{tabular}{lcccc}
\hline Tratamiento & Granulosa & Teca-Granulosa & Tecoma \\
\hline Histerectomía abd. SOB & 7 & 1 & 1 \\
Histerectomía abd. SOB omentectomía & & 1 & 1 \\
SOD, Cuña ovari izq. & -7 & - & 1 & 3 \\
\hline
\end{tabular}

La mayoría de los autores sostienen que el tratamiento de los tumores de la granulosa y teca-granulosa en las pacientes pre-puberales es la salpingooforectomía unilateral y con frecuencia se observa cómo la ciru- 
gía produce una rápida y espectacular regresión de los síntomas y signos de precocidad. En el caso de mujeres jóvenes 0 aquellas en quienes no es posible resolver la duda sobre la malignidad se extirpará solo el tumor y se esperará el informe histopatológico definitivo. En aquellos casos donde el patólogo nos informe sobre la malignidad del tumor se debe reoperar con el objeto de practicar una operación más radical.

Según Benson (3) en los tecomas la salpingooforectomía unilateral casi siempre es el tratamiento para los casos benignos y la histerectomía abdominal total con SOB será el tratamiento para las neoplasias malignas.

Es preferible la biopsia por congelación al tiempo de operar, así el cirujano se hallará en mejores condiciones para tomar una decisión acerca de lo radical que ha de ser la cirugía. Sin embargo no hay que esperar lograr una precisión diagnóstica del $100 \%$ especialmente en tumores disontogénicos donde las imágenes microscópicas no siempre son paralelas a la evolución clínica.

Lo más lógico es que la extensión de la cirugía depende de la integridad de la cápsula, la bilateralidad, edad, paridad y extensión del tumor a los órganos vecinos. "En nuestros casos la intervención más frecuente fue la Histerectomía abdominal con $\mathrm{SOB}$, pues la mayoría se trataba de pacientes multíparas y premenopáusicas donde se justificaba esta conducta; aquellos casos donde se complementó con Omentectomía existía invasión a peritoneo, sigmoides y epiplón o el cirujano procedió en esa forma con el fin de realizar una cirugía más completa. Líquido ascítico se halló en tres casos.

En cuanto al Tecoma en un solo caso se practicó Histerectomía abdo- minal con $\mathrm{SOB}$, pues se trataba de una enferma de 60 años con una hiperplasia adenomatosa. En otro caso además de la Histerectomía abd. con $\mathrm{SOB}$ se le practicó omentectomía, cirugía ésta que estuvo de más si se tiene en cuenta que estos tumores son en su mayoría de carácter benigno. Otro caso se le practicó SOB más cuña de Ovario Izq. en una enferma GO PO con gran anhelo de maternidad, conducta que creemos correcta. Según Grifiths (12) el tratamiento más radical en casos de Tecomas es la salpingooforectomía unilateral y resección en cuña del otro ovario en paciente joven o de edad mediana; si la paciente está en edad premenopáusica o postmenopáusica el tratamiento más lógico es la histerectomía abd. con SOB.

No hay acuerdo en cuanto al uso de la radioterapia postoperatoria, Io más aceptado como opinan Norris y Chorlton (21) es que este procedimiento debe reservarse para aquellos tumores que rebasen el ovario y para los de recurrencias tardías. Según Benson (3) la roentgenoterapia no ha demostrado ser de gran valor en estos tumores $y$ en los tecomas tendrá cabida en aquellos casos con transformaciones malignas.

En nuestro estudio se complementó con irradiación postoperatoria, aquellos donde se encontró invasión a órganos vecinos en el acto quirúrgico.

\section{Pronóstico:}

El pronóstico de los tumores de la granulosa y teca-granulosa depende en realidad de la invasión a los órganos vecinos. Hay que tener presente que el pronóstico de los tecomas es bueno, pues como se dijo anteriormente un gran porcentaje son de carácter benigno. 


\section{Anatomía patológica postoperatoria :}

Leiomioma uterino. Tumor célula de granulosa bilateral.

Tumor célula granulosa derecho, metástasis a epiplón. Adenomatosis.

1 caso

Tumor célula granulosa bilateral. Salpingitis crónica bilateral.

Tumor célula granulosa ovario izquierdo.

Tumor célula granulosa bilateral con invasión ligamento ancho más ca. in situ de cérvix.

1 caso

Tumor célula granulosa ovario derecho con quiste simple ovario, hiperplasia ganduo-quística.

Tumor teca-granuosa izq., quiste seroso ovario derecho más endometrio proiferativo con áreas de hiperpasia adenomatosa y glandu-quística.

1 caso

Tumor teca-granulosa bilateral más hiperplasia glandulo-quística.

1 caso

Tecoma ovario derecho.

1 caso

Tecoma bilateral más cervicitis crónica, más endometrio adenomatoso.

1 caso

Tecoma ovario derecho, más focos de tecomatosis ovario izq. más endometrio secretor más cervicitis crónica.

Griffiths (12) ha llamado la atención en sus trabajos sobre la alta incidencia de leiomiomatosis y de adenomiosis asociados a los teca-granulosos, lo cual justifica hasta cierto punto practicar la histerectomía abdominal.

Como caso interesante encontrados por nosotros, fue la asociación de un tumor de células de granulosa bilateral con invasión al ligamento ancho y ca. in situ de cérvix uterino, el cual se trató por medio de una histerectomía abdominal con SOB.

\section{Controles:}

Es lamentable afirmar que solamente tres enfermas vinieron a control: Una a los 2 años, otra a los 6 años y la última a los 10 años de intervenidas, cuyos controles fueron satisfactorios; las demás no volvieron, lo que nos impide sacar conclusiones sobre tal punto.

\section{Conclusiones:}

1. Se presentan varios conceptos generales donde se afirma que los tumores funcionantes del ovario derivan del estroma gonadal.

2. Se presenta una clasificación propuesta por Norris y Chorlton de los tumores funcionantes del ovario en seis grupos: Tumores del estroma gonadal; Tumores con estroma funcional; Tumores funcionales de células germinativas; Tumores mixtos del estroma y células germinativas; Tumores no endocrinos con efectos endocrinos, Hiperplasias tumorales que simulan neoplasias.

3. Se presenta la frecuencia de estos tumores en general la cual va entre el $3 \%$ al $6 \%$ aproximadamente de todos los tumores ováricos. Particularmente la frecuencia en el Hospital de mujeres de Boston (Estados Unidos), de los tumores de células de la granulosa es de 17,5\%; teca-granulosa $15 \%$; tecomas $67,5 \%$.

4. Se comentan las diferentes teorías sobre la histogénesis de estos tumores.

5. Se hace una descripción sobre su anatomía patológica.

6. En cuanto a la malignidad se refiere, los tumores de la granulosa y los teca-granulosas se consideran que poseen una tasa de malignidad relativamente baja, entre el 3 al $25 \%$; en cambio los tecomas se consideran 
un tumor benigno, pudiendo llegar apenas al $1 \%$.

7. La asociación de estos tumores con ca. endometrial es del 3 al $20 \%$. Parece que el tecoma ejerce efecto carcinógeno más frecuente sobre el endometrio que los demás tumores funcionantes.

8. Se presentan 12 casos de tumores de células de la granulosa, tecagranulosa y tecomas hospitalizados y tratados en el Servicio de Ginecología del Instituto Materno Infantil de Bogotá "Concepción Villaveces de Acosta" en un lapso comprendido entre el 19. de Enero de 1960 y el 30 de Junio de 1975, es decir 15 años y medio.

9. En cuanto a la edad de aparición de estos tumores pueden encontrarse en mortinatos y en mujeres de edad adulta. En los casos por nosotros estudiados la paciente más joven tenía 24 años (tumor de células de granulosa) y la de mayor edad 70 años (tumor teca-granulosa); la edad de los tecomas varió entre los 38 y los 60 años.

10. La sintomatología está dada por la actividad estrogénica del tumor y la época de aparición tumoral. Además de esto hay que tener en cuenta que puede presentar una sintomatología semejante a la de cualquier tumor ovárico: Masa pélvica, dolor pélvico, torsión del tumor.

11. Como diagnósticos preoperatorios hubo los de: miomatosis uterina, quiste de ovario, ca. de ovario. En ninguno de los casos se llegó a un diagnóstico preoperatorio de estos tumores. En aquellos casos donde se practicó raspado uterino se encontró en la patología endometrio atrófico, en otros endometrio proliferativo y en otros endometrio ade- nomatoso. En ningún caso se encontró ca. de endometrio.

12. Generalmente estos tumores son unilaterales.

13. En cuanto al tratamiento creemos al igual que la mayoría de los autores que depende de la invasión del tumor, la bilateralidad, la edad y la paridad de la paciente para así en esa forma practicar una cirugía conservadora o radical. Se ha discutido si la roentgenoterapia postoperatoria es o nó beneficiosa. Creemos que aquellos casos donde se encuentra en el acto quirúrgico invasión, y en las recidivas deban ser erradicadas.

14. El pronóstico de estos tumores depende del grado de invasión tumoral y de la cirugía efectuada. Hay que tener en cuenta que la mayoría de los tecomas son benignos.

15. La anatomía patológica final fue muy variada. Algunos autores llaman la atención sobre la concomitancia de estos tumores con miomatosis y adenomiosis. Fue interesante un caso estudiado por nosotros donde existía la concomitancia del tumor de células de la granulosa con un ca. in situ de cervix uterino.

16. Lamentablemente los controles de estas enfermas fueron muy deficientes, regresaron a control solamente 3 enfermas: una a los 2 años, otra a los 6 años y otra a los 10 años, encontrándose en buenas condiciones. Las demás no volvieron.

\section{Resumen :}

Se presentan 12 casos de tumores de la granulosa, teca-granulosa y tecomas manejados en 15 años.

La edad de aparición entre 24 y 70 años; no se les diagnosticó preoperatoriamente; generalmente unilaterales. 
Se revisan las diferentes teorías etiológicas; se comentan su Histología, su clínica y su tratamiento.

\section{Summary :}

There are presented 12 Granular Tumor cases, Teca-Granular and Tecomas managed during 15 years.

The apparition age was between 24 to 70 years, it was not a Pre-Operatory diagnostic. In general they were unilateral.

The different etiologycal theories are revised. Its histiology, its diagnostic and treatment, are comented.

\section{BIBLIOGRAFIA}

1 ACKERMANN LAUREN VAND DEL REGATO JUAN A. Cáncer. Diagnosis, Treatment and Prognosis 3th Edition The C. V. Mosby Company. St. Louis 1962.

2 ANDERSON W. A. D. Pathology. The C. Mosby Comp. St. Louis 4th edition. Pag. 1096. 1961.

3 BENSON C. RALPH: Manual de Ginecología y Obstetricia. 1a. edición. Editorial El Manual Moderno S. A. 1966.

4 BOTELLA LLUSIA JOSE. Endocrinología de la mujer. Edit. científico médica. Barcelona. 4a. edición, 1966. Pag. 555-561 y 860.

5 BUSBY T. y ANDERSON G. W.: Feminizing mesenchymonas of ovary amer. J. Obstet. Gynec. 68: 1391, 1954.

6 DIDLE A. W.: Granulosa and theca ovarian tumor: Prognosis, cancer 5: 215, 1952.

7 DUARTE CONTRERAS ALBERTO, ZUÑIGA GAMBOA RAMIRO, URIBE BOTERO GONZALO: Tumor de la Granulosa asociado a Adenocarcinoma de Endometrio. Presentación de un caso. Rev. Col. de Obst. y Ginec. Marzo-Abril de 1968. fol. XIX No 2.

8 EMGE LUDWUINS A.: Endometrial Cancer Feminizin tumors of ovaries significance of their coexistence. Am. J. Obst. and Gynec. 1: 511-515. 1953 May.

9 FLICK F. H. y BANFIELD R. S. Jr.: Malignant Theca cell tumors, Cancer 9: 731, 1956.

10 GOODALL C. M.: On para endocrine cancer Syndrome. A. review. Int. J. Cancer. 4; 1, 1969.
11 GOUBERT LAVERDE CARLOS ARTURO: TUmores del Ovario. Rev. Col. de Obst. y Ginec. Mayo-Junio de 1965. Vol. XVI No 3.

12 GRIFFITHS C. THOMAS: Tratado de Ginecología por Kinster Robertm. Ediciones Toray, Barcelona. 1a. edición 1974.

13 HERTIG A. T. y GORE H. M.: Tumore of the female sex organs. Parte 3 tumors of the ovary and fallopian tube Sec IX. fasc. 33 in Atlas of tumor Pathology (Washington, D.C.: Armed Forces Institute of Pathology, 1961).

14 KOTTMEIER H. L.: Moder trends in the treatment of patient with semimalgnant and malignant ovarian tumors in carcinoma of the uterine cervix, endometrium and ovary (Chicago: year book Medical Publishers, Inc. 1962).

15 LOPEZ ESCOBAR GUILLERMO: Tumores del Ovario. Medicine y Cirugía. 8-9-10. 1951.

16 MANSELL H., HERTIG A. T.: Granulosatheca cell tumors and endometrial carcinoma. Obstet. Gynec. 6: 385, 1955.

17 MATZ M. H.: Bening Cystec Teratomas of the ovary, Obst. Gynec. Surg. 16: 591, 1961.

18 MCKAY D. G. HERTIG A. T. Y HICKEY W. F.: The histogenesis of granulosa and theca cell tumors of the human ovary. Obst. \& Gynec. 1: 125. 1953.

19 MEYER R.: Pathology of some special ovarian tumors and their relation to sex characteristics. Am. J. Obst. \& Gynec. 22: 697, 1931.

20 NORRIS H. J.; TAYLOR H. B.: Prognosis of granulosa: theca tumors of the ovary, Cancer 21: 255, 1958.

21 NORRIS J. HENRY y CHORLTON: Tumores funcionales del Ovario. Clínicas Obstétricas y Ginecológicas. Marzo de 1974. Editorial Interamericana.

22 NOVAK EDMUND SEEGAR JONES GEORGEANNA; JONES Jr. HOWARD. Tratado de Ginecología. 7a. edición. Editorial Interamericana.

23 PARSONS and SOMMERS. Gynecology. Ed. W. B. Saunders Comp. Philadelphia and London. Pag. 794, 1968.

24 ROBBINS STANLEL L. Tratado de Patología. Ed. Interamericana S. A. 2a. edición. Pág. 922, 1962.

25 SOMMERS S. C.; GATES O. U GOODOF I. L.: Late recurrence granulosa cell tumors Obstet. Gynec. 6: 1391, 1954. 